\title{
Supervised Parametric and Non-Parametric Classification of Chromosome Images
}

\author{
M. P. Sampat ${ }^{\text {a }}$ A. C. Bovik ${ }^{\text {b J. K. Aggarwal }}{ }^{\text {b }}$ \\ K. R. Castleman ${ }^{\mathrm{c}}$ \\ ${ }^{a}$ Dept. Of Biomedical Engineering, The University of Texas at Austin, TX 78712 \\ ${ }^{\mathrm{b}}$ Dept. Of Electrical and Computer Engineering, The University of Texas at \\ Austin, TX 78712 \\ ${ }^{\mathrm{c}}$ Advanced Digital Imaging Research, LLC, League City, Texas 77573
}

\begin{abstract}
This paper describes a fully automatic chromosome classification algorithm for Multiplex Fluorescence In-Situ Hybridization(M-FISH) images using supervised parametric and non-parametric techniques. M-FISH is a recently developed chromosome imaging method in which each chromosome is labelled with 5 fluors (dyes) and a DNA stain. The classification problem is modelled as a 25-class 6 -feature pixel-bypixel classification task. The 25 classes are the 24 types of human chromosomes and the background, while the six features correspond to the brightness of the dyes at each pixel. Maximum likelihood estimation, nearest neighbor and k-nearest neighbor methods are implemented for the classification. The highest classification accuracy is achieved with the k-nearest neighbor method and $\mathrm{k}=7$ is an optimal value for this classification task.
\end{abstract}

Key words: M-FISH, Nearest Neighbor, k-Nearest Neighbor, Maximum Likelihood Estimation, Karyotyping

\section{Introduction}

Cytogenetics is the study of the genetic makeup of cells. Chromosomes are structures that contain the genetic information of cells. Images of chromosomes taken during cell division contain valuable information about the well being of an individual. Chromosome images are useful for diagnosing genetic disorders and for studying cancer. Thus the analysis of chromosomes is an important procedure in cytogenetic studies.

Preprint submitted to Elsevier Science 18 October 2004 
There are 46 human chromosomes which consist of 22 pairs of similar, homologous chromosomes, and two sex-determinative chromosomes. Thus there are 24 types, or classes, of chromosomes. The process of assigning the the chromosomes to the different classes is known as Karyotyping [1].

Images of chromosomes are analyzed by cytogeneticists to obtain vital information about the health of an individual. However, manual examination of these images is a laborious and time-consuming process and requires skilled lab technicians [2]. Many successful attempts have been made to automate parts of the chromosome image analysis procedure. One of the first steps in chromosome analysis is automated karyotyping.

Images of chromosomes may be obtained using a number of specimen preparation methods. One such method is Multiplex Fluorescence In-Situ Hybridization (M-FISH) $[3,4]$ which is a recently developed chromosome imaging technique. The goal of the research described in this paper is the automated classification of chromosome images that have been obtained by M-FISH.

The first paper on the M-FISH technique was published in 1996 by Speicher et al. [3] and it revolutionized chromosome imaging. In this technique chromosomes are labelled with five fluors (dyes) and a fluorescent DNA stain called DAPI (4',6-Diamidino-2-phenylindole).

DAPI attaches to DNA and thus labels all chromosomes. The fluors attach to specific sequences of DNA. With M-FISH a unique combination of fluors is assigned to each chromosome type. That is, each class of chromosomes absorbs a different combination of fluors[3]. Thus M-FISH is based on a combinatorial labelling strategy. This strategy provides an easy way to label chromosomes in a multiplex fashion, as each fluor is either present(1) or absent(0) [3,5]. Also, at least five distinguishable fluors are needed for combinatorial labelling to uniquely identify all 24 chromosome types as the number of useful combinations of $N$ fluors is $2^{N}-1[3,5]$.

The central idea in M-FISH is that each chromosome is labelled by a unique combination of the five fluors. Several such sets of fluors have been developed for M-FISH imaging. One such set of five fluors and the corresponding fluor labelling table is shown in Table 1 [6]. The fluor labelling table enumerates the different combinations of the fluors used to label each chromosome type.

Though in theory the fluor absorption is described as binary, this is not the case in practice for real M-FISH data-sets [7].

M-FISH images are captured with a fluorescent microscope. Multiple optical filters are used to view each of the fluorescent fluors. Each of the fluors is visible in one of the spectral channels. Thus a set of M-FISH images can be viewed as a multi-spectral set. An M-FISH data set consists of six images 
where each image is the response of the chromosome to a particular fluor. A typical M-FISH data set is shown in Figure 1. Figures 1(a) to 1(e) are the images of the responses of the five fluors which are Spectrum Aqua, Far Red, Spectrum Green, Spectrum Red and Spectrum Gold, respectively [6]. Figure 1 (f) shows the response of the DNA stain DAPI. DAPI attaches to DNA and thus all chromosomes are seen in this image.

Semi-automated image analysis of M-FISH data was done by Speicher et al. [5] in 1996. This basically consisted of segmentation, thresholding and classification stages. The DAPI channel was used to create a mask to segment the chromosomes from the background. This mask and a threshold were applied to each M-FISH image to detect the presence or absence of a fluor at each pixel. Each pixel was then classified by comparing the combined response of the fluors at that pixel to the combinations in a fluor labelling table.

The image analysis was fully automated by Elis et al.[8] in 1998. They modelled the task as a 5-feature 24-class pattern recognition problem and performed adaptive spectral analysis for classification. This consisted of spectral calibration and adaptive region-oriented classification. During the calibration step an optimal vector to represent each class was found by minimizing an energy term. These vectors were called adaptive spectral feature vectors. In the classification step the image was subdivided into various polygons using Voronoi tessellation. The closest adaptive spectral feature vector (spectral class) for each region was computed. These were then classified using an iterative region-growing algorithm. Regions with color vectors best approximating the adaptive spectral feature vectors were used as the starting points for the region-growing process. Two regions were merged if they belonged to the same class and the merged region was assigned the class of the start region. They claim that pixel-by-pixel classification would produce noisy results and thus did not perform pixel-by-pixel classification[8].

Saracoglu et al. [9] modelled the problem similarly. Their algorithm consisted of three steps, image tessellation, clustering and classification. The image was tessellated into regions with similar properties with a region-growing algorithm. Then an average color vector was computed for each region. For each of the classes, one start vector was selected (from the set of color vectors) such that it was the closest vector to the theoretically optimal color class vector. These 24 start vectors were then used as starting points for a k-means clustering algorithm. Each cluster was then classified by comparing its centroid with the theoretical color class vectors. However, none of these papers reported the classification accuracies of their methods over various M-FISH image sets.

In this paper we propose new algorithms for pixel-by-pixel classification of M-FISH images and show that this methodology gives good results. In these algorithms we use all six images of the M-FISH data set and we include the background as a new class. Thus we have modelled the problem as a 6-feature 25-class pattern recognition task. We report the classification accuracies of the 
method over various M-FISH data sets.

The rest of the paper is organized as follows. Section Two describes the different classification techniques. The methodology and the data sets used are described in Section Three. The results are presented in Section Four. Finally, Section Five presents the conclusion.

\section{Classification Techniques}

This section gives a brief review of the different supervised parametric and non-parametric classification techniques that are used in this paper. The aim of these techniques is to classify samples into one of $N$ different classes based on features that describe the sample. Let $w_{i}$ for $i=1, \ldots, N$ denote the $N$ classes. If we measure $d$ features for each sample then each sample is described by a d-dimensional feature vector. Let $x$ denote such a feature vector. A classifier is first trained on a given labelled set of training samples. A given test sample is then assigned to a particular class by the classifier. The details of the different classifiers are described below[10].

\subsection{Supervised Parametric Method}

The supervised parametric method used is maximum likelihood estimation. Let $P\left(w_{i}\right)$ denote the a priori probability that a sample belongs to class $w_{i}$ where $i=1, \ldots, N$.

Let $p\left(x \mid w_{i}\right)$ denote the class-conditional probability density function. It represents the probability distribution function for a feature vector $x$ given that $x$ belongs to class $w_{i}$. Let $P\left(w_{i} \mid x\right)$ be the aposteriori probability, which is the probability that the sample belongs to class $w_{i}$ given the feature vector $x$. Given $P\left(w_{i}\right)$ and $p\left(x \mid w_{i}\right)$, the a posteriori probability for a sample represented by the feature vector $x$ is given by the Bayes formula [10].

$$
P\left(w_{i} \mid x\right)=\frac{p\left(x \mid w_{i}\right) P\left(w_{i}\right)}{p(x)}
$$

where $p(x)=\sum_{i=1}^{N} p\left(x \mid w_{i}\right) P\left(w_{i}\right)$. The formula is applicable for all probability density functions; however, depending on the nature of the data, the normal density function is often used to model the distribution of feature values of a particular class. The general multivariate normal density function in $d$ dimen- 
sions is given by:

$$
p(x)=\frac{1}{(2 \pi)^{d / 2}\left|\sum\right|^{1 / 2}} \exp \left[-\frac{1}{2}(x-\mu)^{t} \sum^{-1}(x-\mu)\right]
$$

where $x$ is a $d$ component feature vector, $\mu$ is the $d$ component mean vector, $\sum$ is the $d \times d$ covariance matrix, and $\left|\sum\right|$ and $\sum^{-1}$ are its determinant and inverse, respectively. It is assumed that the density function for each class is a 6 -dimensional Gaussian function. The parameters $\mu$ and $\sum$ of the probability density function for each class are calculated from the training samples belonging to that class. Note that the maximum likelihood estimates for $\mu$ and $\sum$ of each class are the mean vector and covariance matrix of the training samples of that class. Any given test sample, described by the feature vector $\mathrm{x}$, can be classified by using the Bayes Decision Rule, which is:

$$
\text { decide } w_{i} \text { if } P\left(w_{i} \mid x\right)>P\left(w_{j} \mid x\right) \forall j \neq i
$$

\subsection{Supervised Non-Parametric Methods}

The supervised non-parametric methods selected for classification are the nearest neighbor and the k-nearest neighbor methods. In these methods no assumptions are made about the probability density function for each class. These methods are used because the assumption that the probability density function for each class is a 6-dimensional normal distribution may not necessarily be true, and a classifier may perform better if these assumptions are not made.

\subsubsection{Nearest Neighbor}

Let $T=\left\{s_{1}, s_{2}, \ldots, s_{n}\right\}$ denote the set of $n$-labelled training samples. Each sample is a $d$-dimensional vector. Let $s_{i} \in T$ be the training sample nearest to a given test sample $t$ in terms of some metric or distance function. The nearest neighbor rule for classifying $t$ is to assign it to the class to which $s_{i}$ belongs [10]. The metric we use is the Euclidean distance.

\subsection{2 k-Nearest Neighbor}

Let $T=\left\{s_{1}, s_{2}, \ldots, s_{n}\right\}$ denote the set of $n$-labelled training samples. Given a test sample $t$, let $R=\left\{r_{1}, r_{2}, \ldots, r_{k}\right\}$ be a set of the $k$-nearest training samples to $t$ in terms of some metric. The $k$-nearest neighbor rule is to assign the sample $t$ to the class that occurs most frequently among the $k$-nearest training samples. Again the metric used is the Euclidean distance. The values

of $k$ used are 5,7 and 9 neighbors. If the ranges of the data in each dimension 
vary considerably, this may affect the performance of the nearest neighbor and $k$-nearest neighbor drastically. Thus both the training and testing data must be normalized. We used the following method for normalization of the data.

$$
y=(x-\mu) /(3 * \sigma)
$$

where $x$ is the $d$-dimensional original data sample, $\mu$ is the $d$-dimensional mean vector of the given training samples, $\sigma$ is the standard deviation of the training samples, and $y$ is the normalized data sample.

\section{Methodology}

The supervised parametric and non-parametric methods described in Section 2 were used for classification. For all of the methods, we used the same training and testing samples so that a fair comparison could be made between them. To compare the performance of the two methods, the overall classification accuracy and the chromosome classification accuracy were measured. The chromosome classification accuracy is the accuracy of classifying only those pixels belonging to chromosomes. Since a majority of the pixels are background pixels, the overall pixel classification accuracy mainly reflects segmentation. Thus, it is important to measure the chromosome classification accuracy to get a good idea of the diagnostic performance of the classifier.

The images for training and testing were selected from a public database of M-FISH images. This database is made available online by Advanced Digital Imaging Research and can be accessed at:

http : //www.adires.com/05/Project/MFISH_DB/MFISH_DB.shtml.

For each set of M-FISH images the database also contains a labelled class-map image in which each pixel is labelled according to the class to which it actually belongs. This image was used to determine the accuracy of the different classification techniques.

For training, pixels belonging to each of the classes were chosen randomly ten times, from one set of M-FISH images. Thus ten different training data sets were created. Pixels from other sets of M-FISH images were chosen for testing. Thus there was no overlap between the training and testing data. Each set of testing data was then classified with respect to each of the training data sets. The classification results (the overall accuracy and the chromosome accuracy) obtained from the ten trials were then averaged to obtain the final classification results for each test set. This was done for each classification method and for every test set. Since $90 \%$ or more of the pixels of each M-FISH set were background pixels, only a subset of pixels from each set were selected for testing. The selection of pixels for testing is described in Section 3.1. 


\subsection{Selection of Pixels for Classification}

The goal was to create a binary image(mask) in which the pixels to be selected for testing are labelled "1" whereas the pixels not to be selected are labelled "0". As mentioned before, the DAPI stain labels all of the chromosomes, and thus the image of the DAPI channel was used for the selection of pixels. This image is shown in Figure 2(a). First the edges of the chromosomes in the DAPI image were detected using the Laplacian of Gaussian edge detector. Figure 2(b) shows the edges detected. A review of this method appears in $[11,12]$. The edge image was then dilated using a morphological operator, as shown in Figure 2(c). This was done because perfect segmentation of the chromosomes is difficult to achieve and it was seen that some faint pixels belonging to some chromosomes fell outside the edges detected. Dilation ensured that these pixels were also included in the classification stage. Finally all pixels lying inside the edges of the chromosomes were set to 1 , and those lying outside were set to 0 to create the mask shown in Figure 2(d). The boundaries of the objects in Figure 2(d) were detected and overlaid on the original image in Figure 2(e).

\subsection{Classification and Post-Processing}

The pixels selected by the process described in Section 3.1 were classified by maximum likelihood estimation(MLE), nearest neighbor(NN) and k-nearest neighbor $(\mathrm{k}=5,7$ and 9) classifiers. Before training, all pixels were first normalized by the procedure described in Section 2.2.2. All of these classifiers were then trained with the same set of training samples. A class-map for each output was generated. In this image each pixel was labelled according to the class it was classified to.

Isolated pixel classification errors were observed after the classification. To remove these errors, a 5-by-5 majority filter was applied to the classification output. In majority filtering, an n-by-n window is centered about each pixel in a given image. The value that occurs the maximum number of times among the values lying within the window is determined. This output is placed at the location of the center pixel, that is, the pixel about which the window was centered. This procedure is then repeated for every pixel in the image. Majority filtering significantly improve the classification accuracy.

\section{Results}

Five M-FISH image sets, labelled A to E, were classified using the methods described above. Each set has 333, 465 pixels. From each of these, a subset of 
pixels was selected for testing by applying the pixel selection algorithm described in Section 3.1. For each set, the average overall classification accuracy and the average chromosome classification accuracy were computed. A classmap was generated for each classification output. A separate color was used to represent each chromosome class in the image. The overall and chromosome accuracies were computed by comparing this class-map to the class-map provided in the database.

Tables 2 and 3 show the chromosome classification accuracy and the overall classification accuracy obtained for each M-FISH set without application of the majority filter. Tables 4 and 5 show the chromosome classification accuracy and the overall classification accuracy obtained after application of the majority filter to the classification result. Majority filtering improves classification accuracy by reducing the number of isolated pixel classification errors. It reduced the average chromosome misclassification rate by $2 \%$.

Figure 3 shows the classification results for the M-FISH Image Set A. The actual class-map is shown in Figure 3(a) and the computed class-maps before and after majority filtering are shown in figures 3(b) and 3(c) respectively. Similarly, the results for the other M-FISH image sets (B to E) are shown in figures 4 to 7 , respectively. These figures show the results obtained with the $\mathrm{k}$-nearest neighbor method $(\mathrm{k}=7)$. Figure 8 shows the different classification results for M-FISH Image Set B, obtained with the MLE, NN and k-NN $(k=7)$ classifiers.

A 25 by 25 confusion matrix for one of the classified outputs is shown in Table

6 . The rows and columns of this table correspond to the actual and predicted classes. The first row and column correspond to the class numbers. In this matrix, class 0 corresponds to the background and thus a maximum number of pixels fall in the $(0,0)$ square. Note that most of the entries of this matrix are zeros.

The non-parametric methods give higher classification accuracies than the parametric method. The k-nearest neighbor method outperformed the maximum likelihood and nearest neighbor methods. As the value of $\mathrm{k}$ was increased, the classification accuracy increased. However, we observe very little improvement in accuracy as $\mathrm{k}$ was increased from 7 to 9 and beyond. Thus increasing $\mathrm{k}$ beyond 7 is not beneficial.

\section{Conclusion}

In this paper we have developed new, fully automated algorithms for pixelby-pixel classification of M-FISH images and showed that high classification accuracies can be achieved with this methodology. The overall classification accuracy achieved is $98.3 \%$ and the overall chromosome classification accuracy achieved is $90.52 \%$. 
The classification task is modelled as a 6-feature, 25-class classification problem. Supervised parametric and non-parametric techniques were implemented, and it was found that the Non-Parametric methods performed better than the parametric method. The highest classification accuracy was obtained by the $\mathrm{k}$-nearest neighbor method, and $\mathrm{k}=7$ is an optimal value for this classification task. We also showed that post-processing techniques such as majority filtering can help improve the classification accuracy. 


\section{References}

[1] R. S. Verma, A. Babu, Human Chromosomes: Principles and Techniques, 2nd Edition, McGraw-Hill, Inc., 1995.

[2] Q. Wu, K. Castleman, Automated chromosome classification using waveletbased band pattern descriptors, Proc. of the 13th IEEE Symposium on Computer-Based Medical Systems (2000) 189-194.

[3] M. Speicher, S. Ballard, D. Ward, Karyotyping Human Chromosomes by Combinatorial Multi-fluor FISH, Nature Genetics 12 (1996) 368-375.

[4] M. Beau, One FISH, two FISH, red FISH, blue FISH, Nature Genetics 12 (1996) $341-344$.

[5] M. Speicher, S. Ballard, D. Ward, Computer Image Analysis of Combinatorial Multi-fluor FISH, Bioimaging 4 (1996) 52-64.

[6] W. Schwartzkopf, ADIR M-FISH Image Database, Tech. rep., Advanced Digital Imaging Research (August 2000).

[7] W. Schwartzkopf, Maximum Likelihood Techniques for Joint SegmentationClassification of Multi-spectral Chromosome Images, Ph.D. thesis, The University of Texas at Austin (December 2002).

[8] R. Eils, S. Uhrig, K. Saracoglu, K. Satzler, A. Bolzer, I. Petersen, J. Chassery, M. Ganser, M. Speicher, An optimized, fully automated system for fast and accurate identification of chromosomal rearrangements by multiplex-fish (mfish), Cytogenet. Cell Genet. 82 (1998) 160-171.

[9] K. Saracoglu, J. Brown, L. Kearney, S. Uhrig, J. Azofeifa, C. Fauth, M. Speicher, R. Eils, New concepts to improve resolution and sensitivity of molecular cytogenetic diagnostics by multicolor fluorescence in situ hybridization, Cytometry 44 (2001) 7-15.

[10] R. O. Duda, P. E. Hart, D. G. Stork, Pattern Classification, 2nd Edition, WileyInterscience, San Diego, 2001.

[11] A. C. Bovik, Handbook of Image and Video Engineering, Academic Press, 2000.

[12] K. R. Castleman, Digital Image Processing, Prentice-Hall, 1996.

[13] A. Carothers, J. Piper, Computer-aided classification of human chromosomes: A review, Statistics and Computing 4 (3) (1994) 161-171.

[14] W. Schwartzkopf, B. L. Evans, A. Bovik, Minimum entropy segmentation applied to multi-spectral chromosome images, Proc. IEEE Int. Conf. on Image Processing II (2001) 865-868.

[15] K. Castleman, Digital imaging and cytogenetics a historical perspective, Proc. of the 13th IEEE Symposium on Computer-Based Medical Systems . 
[16] K. R. Castleman, R. Elis, L. Morrison, J. Piper, K. Saracoglu, M. A. Schulze, Classification accuracy in multiple color fluorescence imaging microscopy, Cytometry 41 (2000) 139-147.

ChromosomeSpectrum Aqua|Spectrum Green|Spectrum GoldSpectrum RedFar Red

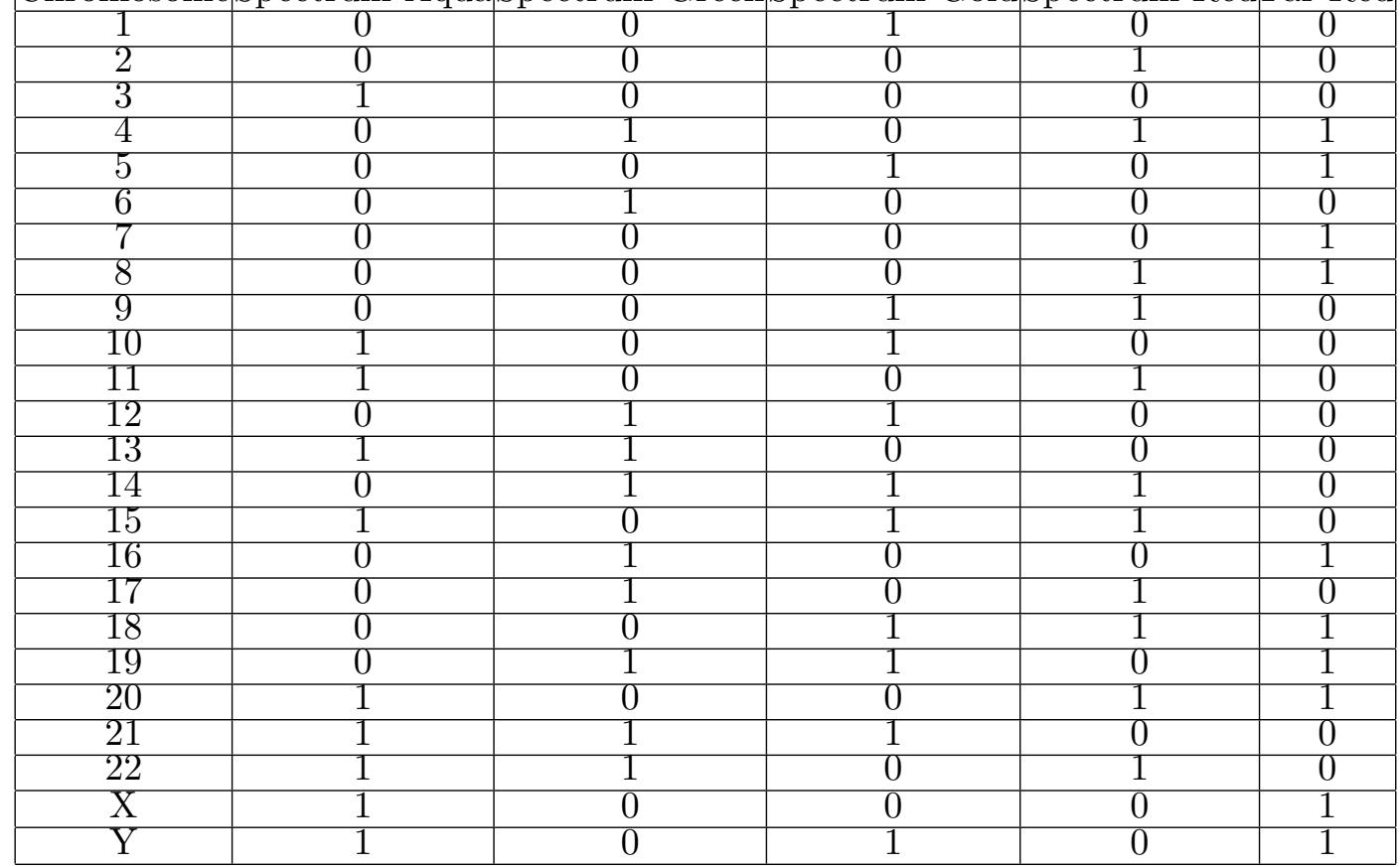

Table 1

M-FISH fluor labelling table: The first column represents the chromosome number. Names of the five different fluors are shown in the first row. A 1 indicates that a particular chromosome is labelled by the fluor and a 0 indicates that the chromosome is not labelled by the fluor. Thus each chromosome is labelled by a specific combination of dyes.

\begin{tabular}{|c|c|c|c|c|c|}
\hline Test Set & MLE & NN & k-NN $(\mathrm{k}=5)$ & $\mathrm{k}-\mathrm{NN}(\mathrm{k}=7)$ & $\mathrm{k}-\mathrm{NN}(\mathrm{k}=9)$ \\
\hline A & 86.2870 & 87.6290 & 88.6620 & 88.7460 & 88.8040 \\
\hline B & 88.3080 & 90.8400 & 92.2720 & 92.6190 & 92.8190 \\
\hline C & 72.3810 & 85.9460 & 87.6780 & 88.0970 & 88.3080 \\
\hline D & 68.0510 & 82.9520 & 85.3300 & 85.8610 & 86.1830 \\
\hline E & 86.5690 & 84.5900 & 85.8430 & 85.9970 & 85.9990 \\
\hline
\end{tabular}

Table 2

Overall chromosome classification accuracy for the different methods without majority filtering. All results in percentages. 


\begin{tabular}{|c|c|c|c|c|c|}
\hline Test Set & MLE & NN & k-NN $(\mathrm{k}=5)$ & $\mathrm{k}-\mathrm{NN}(\mathrm{k}=7)$ & $\mathrm{k}-\mathrm{NN}(\mathrm{k}=9)$ \\
\hline A & 97.3970 & 97.7030 & 97.7700 & 97.7610 & 97.7710 \\
\hline B & 98.2480 & 98.5350 & 98.5630 & 98.5720 & 98.5790 \\
\hline C & 97.1210 & 98.0890 & 98.1380 & 98.1500 & 98.1630 \\
\hline D & 96.3540 & 97.6560 & 97.8240 & 97.8580 & 97.8860 \\
\hline E & 97.8780 & 98.2680 & 98.3180 & 98.3220 & 98.3220 \\
\hline
\end{tabular}

Table 3

Overall classification accuracy for the different methods without majority filtering. All results in percentages.

\begin{tabular}{|c|c|c|c|c|c|}
\hline Test Set & MLE & NN & k-NN $(\mathrm{k}=5)$ & $\mathrm{k}-\mathrm{NN}(\mathrm{k}=7)$ & $\mathrm{k}-\mathrm{NN}(\mathrm{k}=9)$ \\
\hline A & 90.0180 & 90.9640 & 91.2200 & 91.1500 & 91.1270 \\
\hline B & 90.9570 & 93.4560 & 94.2710 & 94.4070 & 94.4690 \\
\hline C & 74.5680 & 89.8400 & 90.5340 & 90.7760 & 90.8470 \\
\hline D & 70.7780 & 87.7670 & 88.8210 & 89.0610 & 89.1680 \\
\hline E & 88.4740 & 86.4730 & 87.0830 & 87.2130 & 87.1190 \\
\hline
\end{tabular}

\section{Table 4}

Overall chromosome classification accuracy for the different methods with majority filtering. All results in percentages.

\begin{tabular}{|c|c|c|c|c|c|}
\hline Test Set & MLE & NN & k-NN $(\mathrm{k}=5)$ & $\mathrm{k}-\mathrm{NN}(\mathrm{k}=7)$ & $\mathrm{k}-\mathrm{NN}(\mathrm{k}=9)$ \\
\hline A & 97.7660 & 98.0410 & 98.0130 & 97.9880 & 97.9900 \\
\hline B & 98.4090 & 98.6960 & 98.6770 & 98.6710 & 98.6700 \\
\hline C & 97.3190 & 98.3910 & 98.3490 & 98.3470 & 98.3500 \\
\hline D & 96.6040 & 98.0640 & 98.0940 & 98.1000 & 98.1100 \\
\hline E & 98.0650 & 98.4360 & 98.4220 & 98.4220 & 98.4120 \\
\hline
\end{tabular}

Table 5

Overall classification accuracy for the different methods with majority filtering. All results in percentages. 


\begin{tabular}{|c|c|c|c|c|c|c|c|c|c|c|c|c|c|c|c|c|c|c|c|c|c|c|c|c|c|}
\hline & 0 & 1 & 2 & 3 & 4 & 5 & 6 & 7 & 8 & 9 & 10 & 11 & 12 & 13 & 14 & 15 & 16 & 17 & 18 & 19 & 20 & 21 & 22 & 23 & 24 \\
\hline 0 & $31211 !$ & 13 & 11 & 0 & 3 & 1 & 8 & 4 & 6 & 0 & 0 & 0 & \begin{tabular}{|l|l|}
0 \\
\end{tabular} & \begin{tabular}{|l|}
10 \\
\end{tabular} & 3 & 9 & 3 & 2 & 0 & 6 & 3 & 0 & & 0 & \\
\hline \begin{tabular}{|l|}
1 \\
\end{tabular} & 220 & 1373 & 0 & 0 & 8 & 2 & 0 & 0 & 8 & 0 & 0 & 0 & 0 & 0 & 1 & 0 & 0 & 0 & 0 & 0 & 0 & 0 & 0 & 0 & 0 \\
\hline \begin{tabular}{|l|} 
\\
\end{tabular} & 118 & 0 & 1361 & 0 & 3 & 0 & 0 & 0 & 0 & 0 & 0 & 0 & 0 & 0 & 1 & 4 & 0 & 0 & 0 & 0 & 0 & 0 & 0 & 0 & 0 \\
\hline 3 & 249 & 0 & 0 & 1058 & 0 & 0 & 0 & 0 & 0 & 0 & 0 & 0 & 0 & 0 & 0 & 0 & 0 & 0 & 0 & 0 & 0 & 0 & 0 & 0 & 0 \\
\hline 4 & 92 & 0 & 16 & 0 & 1018 & 0 & 20 & 0 & 0 & 0 & 0 & 0 & 15 & 0 & 0 & 0 & 0 & 0 & 0 & 1 & 0 & 0 & 0 & 0 & 0 \\
\hline 5 & 101 & 2 & 0 & 0 & 0 & 996 & 0 & 1 & 0 & 0 & 0 & 0 & 0 & 0 & 0 & 0 & 0 & 0 & 0 & 0 & 0 & 0 & 0 & 0 & 0 \\
\hline 6 & 155 & 0 & 0 & 0 & 6 & 0 & 995 & 0 & 0 & 0 & 0 & 0 & 0 & 0 & 0 & 0 & 0 & 0 & 0 & 0 & 0 & 0 & 0 & 0 & 0 \\
\hline 7 & 206 & 0 & 0 & 0 & O & 4 & 0 & 884 & 23 & 0 & 0 & 0 & 0 & 0 & 0 & 0 & 0 & 0 & 0 & 0 & 0 & 0 & 0 & 0 & 0 \\
\hline 8 & 49 & 0 & 6 & 0 & 0 & 0 & 0 & 0 & 753 & 0 & 0 & 0 & 0 & 0 & 0 & 0 & 0 & 0 & 23 & 0 & 0 & 0 & 0 & 0 & 0 \\
\hline 9 & 221 & 0 & 9 & 0 & 0 & 0 & 0 & 0 & 0 & 730 & 0 & 0 & 0 & 0 & 0 & 0 & 0 & 0 & 0 & 0 & 0 & 0 & 0 & 0 & 0 \\
\hline 10 & 192 & 0 & 0 & 0 & 1 & 0 & 0 & 0 & 0 & 0 & 810 & 0 & 0 & 0 & 0 & 0 & 15 & 1 & 0 & 0 & 0 & 0 & 0 & 0 & 0 \\
\hline 11 & 357 & 0 & 0 & 2 & 0 & 0 & 0 & 0 & 0 & 0 & 0 & 775 & 0 & 0 & 0 & 10 & 0 & 0 & 0 & 0 & 0 & 0 & 2 & 0 & 0 \\
\hline 12 & 162 & 3 & 0 & 0 & 0 & 0 & 0 & 0 & 0 & 0 & 0 & 0 & 728 & 0 & 11 & 0 & 0 & 0 & 0 & 0 & 0 & 0 & 0 & 0 & 0 \\
\hline 13 & 85 & 10 & $T$ & 0 & 1 & 0 & 0 & 0 & 1 & 0 & 0 & 0 & 0 & 705 & 0 & 2 & $T$ & 0 & 0 & 0 & 0 & 0 & 0 & 0 & 0 \\
\hline 14 & 85 & 7 & 24 & 0 & 17 & 0 & 0 & 0 & 0 & 0 & 4 & 0 & 35 & 0 & 451 & 1 & 0 & 0 & 0 & 0 & 0 & 0 & 0 & 0 & 0 \\
\hline 15 & 115 & 4 & 0 & 0 & 0 & 0 & 0 & 0 & 0 & 0 & 0 & 0 & 0 & 11 & 0 & 494 & 0 & 0 & 0 & 0 & 0 & 4 & 0 & 0 & 0 \\
\hline 16 & 201 & 1 & 0 & 0 & 36 & 11 & 61 & 7 & 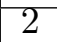 & 0 & 1 & 0 & 0 & 0 & 0 & 0 & 417 & 4 & 0 & 35 & 0 & 0 & 0 & 0 & 0 \\
\hline 17 & 111 & 0 & 0 & 0 & 2 & 0 & 0 & 0 & 0 & 0 & 0 & 0 & 0 & 0 & 0 & 0 & 0 & 512 & 0 & 1 & 0 & 0 & 0 & 0 & 0 \\
\hline 18 & 226 & 0 & 2 & 0 & 0 & 21 & 0 & 0 & 62 & 0 & 0 & 0 & 0 & 0 & 0 & 0 & 0 & 0 & 496 & 0 & 4 & 0 & 0 & 0 & 0 \\
\hline 19 & 115 & 3 & 0 & 0 & 5 & 4 & 2 & 0 & 1 & 0 & 6 & 0 & 20 & 0 & 0 & 0 & 12 & 13 & 0 & 277 & 0 & 0 & 0 & 0 & 0 \\
\hline 20 & 112 & 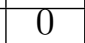 & 0 & 0 & 0 & 0 & 0 & 0 & 2 & 0 & 0 & 4 & 0 & 0 & 0 & 0 & 0 & 0 & 17 & 0 & 348 & 0 & 0 & 0 & 0 \\
\hline 21 & 194 & 0 & 0 & 0 & 3 & 0 & 0 & 0 & 0 & 0 & 5 & 0 & 2 & 31 & 12 & 5 & 3 & 0 & 0 & 0 & 0 & 328 & 14 & 0 & 0 \\
\hline 22 & 148 & 0 & 9 & 0 & 38 & 0 & 0 & 0 & 0 & 0 & 0 & 0 & 7 & 9 & 44 & 2 & 0 & 1 & 0 & 0 & 0 & 0 & 224 & 0 & 0 \\
\hline 23 & 125 & , & 0 & 1 & 0 & 0 & 0 & 0 & 0 & 0 & 0 & 0 & 0 & 0 & 0 & 0 & 0 & 0 & 0 & 0 & 1 & 0 & 0 & 401 & 0 \\
\hline 24 & 67 & 0 & 0 & 0 & 0 & 0 & 0 & 0 & 0 & 0 & 1 & 0 & 0 & 0 & 0 & 10 & 18 & 0 & 0 & 0 & 0 & 0 & 0 & & 253 \\
\hline
\end{tabular}

Table 6

The 25-by-25 confusion matrix for M-FISH Image Set B. The columns correspond to the actual classes and the rows correspond to the predicted classes. Class 0 corresponds to the background. Class 1 corresponds to chromosome 1, and so on. The first row and columns represent the class numbers. 


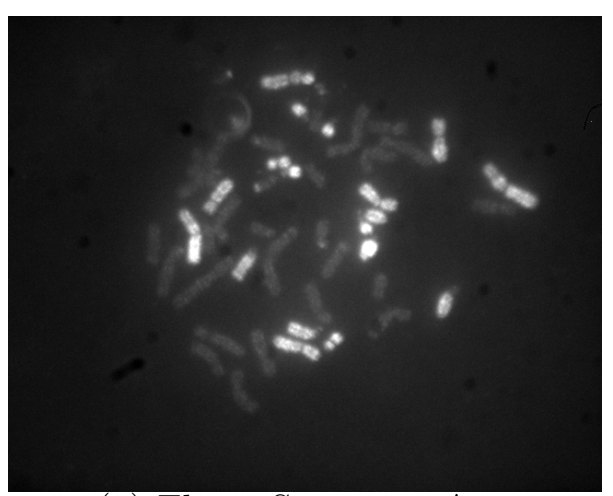

(a) Fluor: Spectrum Aqua

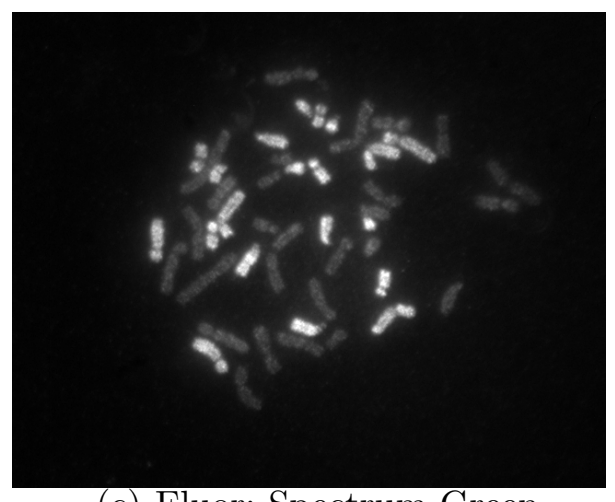

(c) Fluor: Spectrum Green

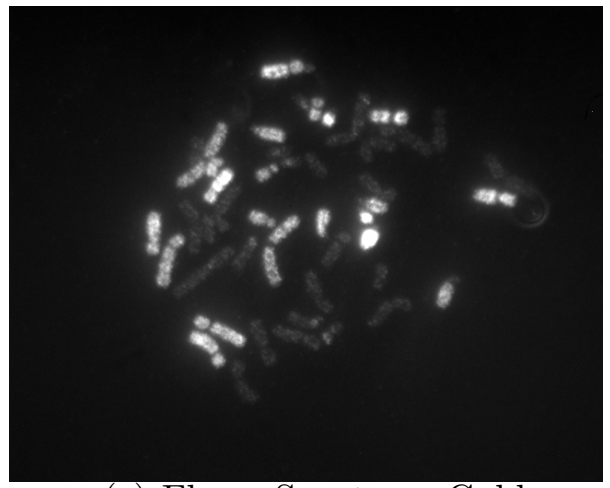

(e) Fluor: Spectrum Gold

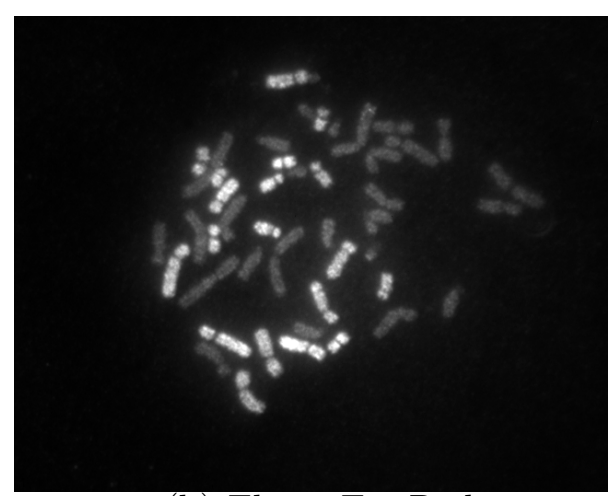

(b) Fluor: Far Red

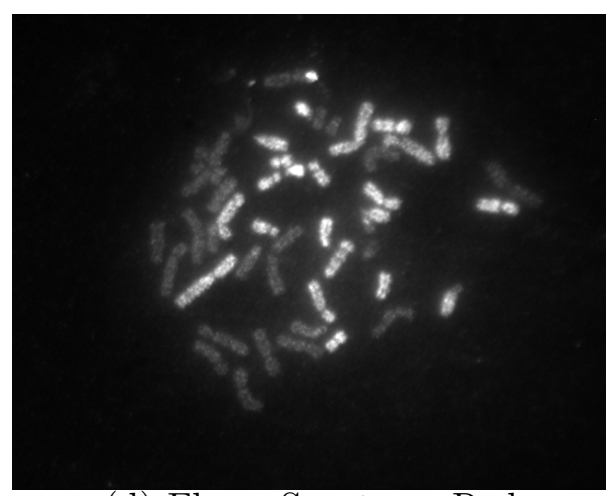

(d) Fluor: Spectrum Red

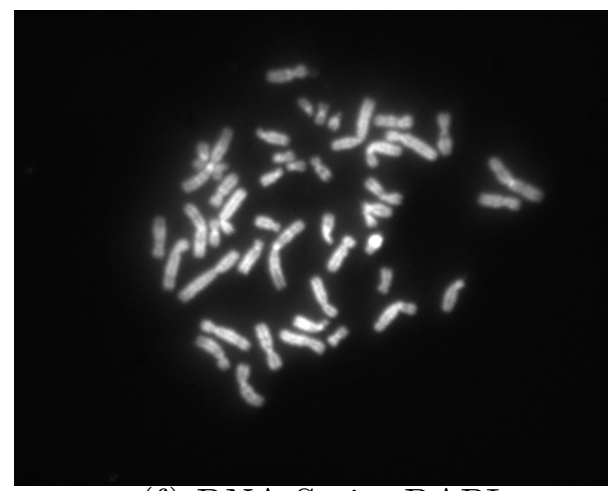

(f) DNA Stain: DAPI

Fig. 1. A set of M-FISH Images. Each image corresponds to the response of a particular fluor. The DAPI stain labels all chromosomes. 


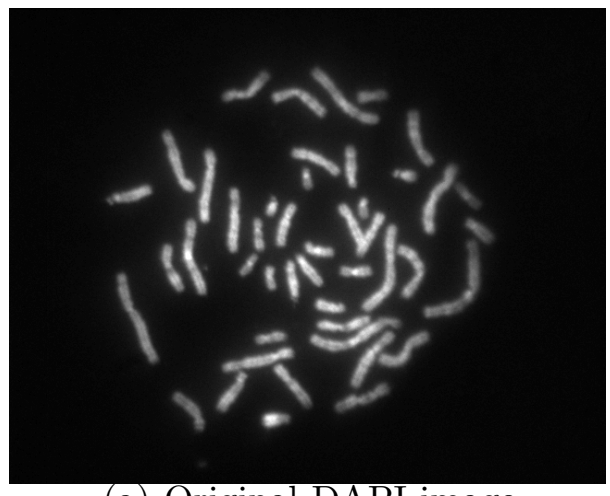

(a) Original DAPI image

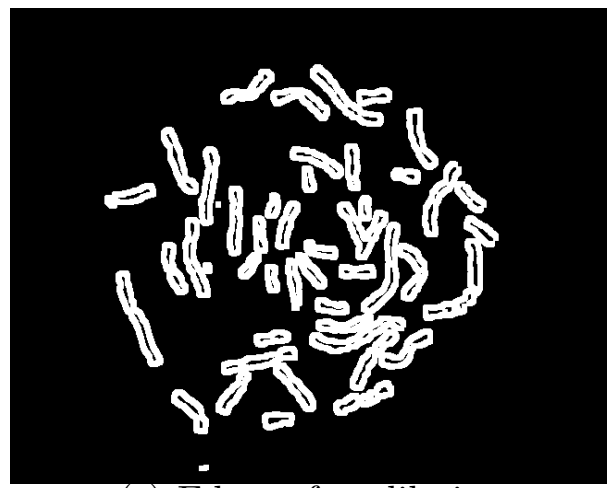

(c) Edges after dilation

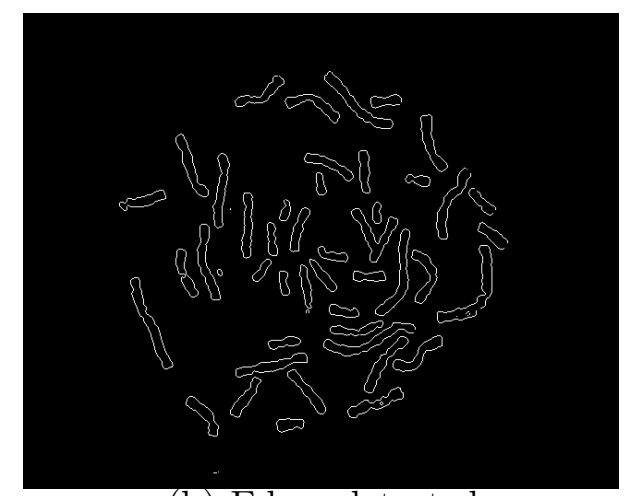

(b) Edges detected

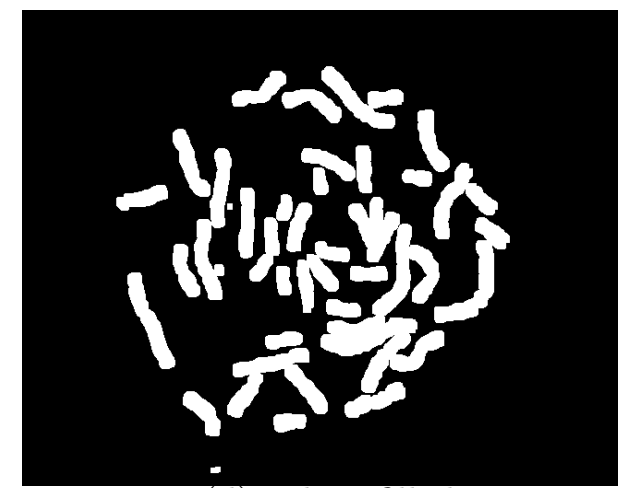

(d) Edges filled

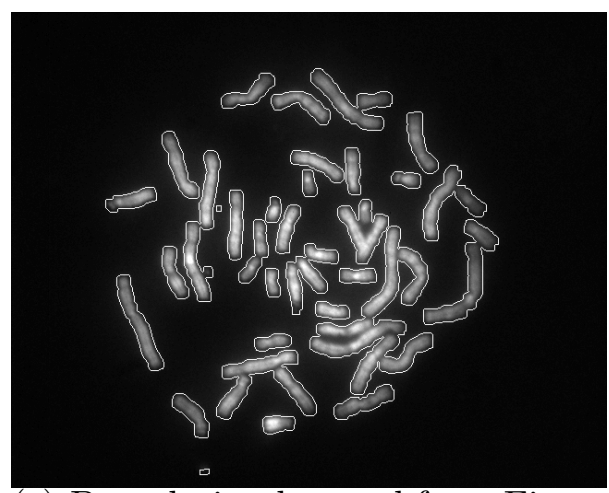

(e) Boundaries detected from Figure

2 (d) overlaid on the original image

Fig. 2. Selection of testing pixels for classification 


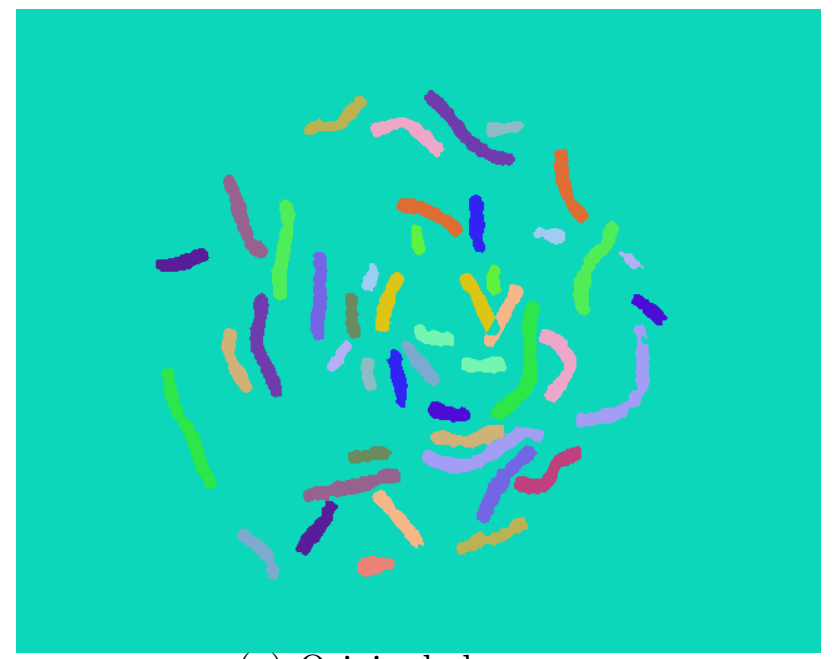

(a) Original class-map
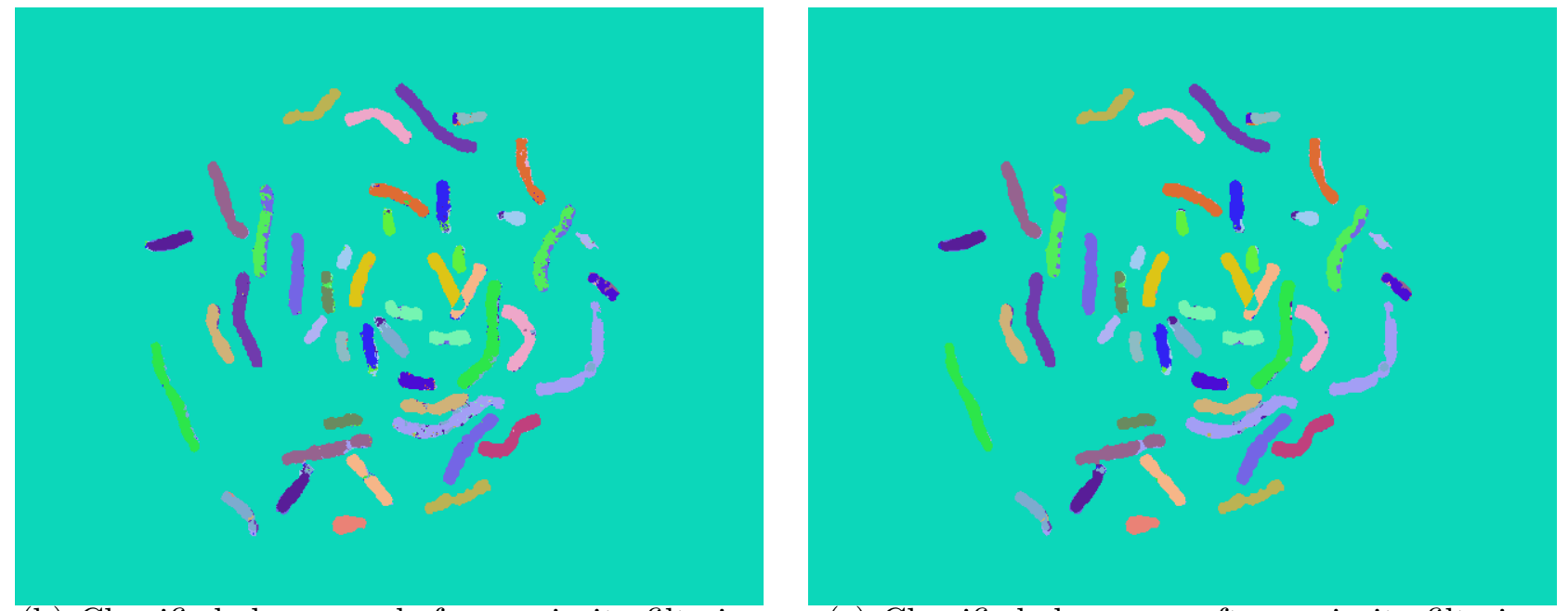

(b) Classified class-map before majority filtering

(c) Classified class-map after majority filtering

Fig. 3. Classification results for M-FISH Image Set A 


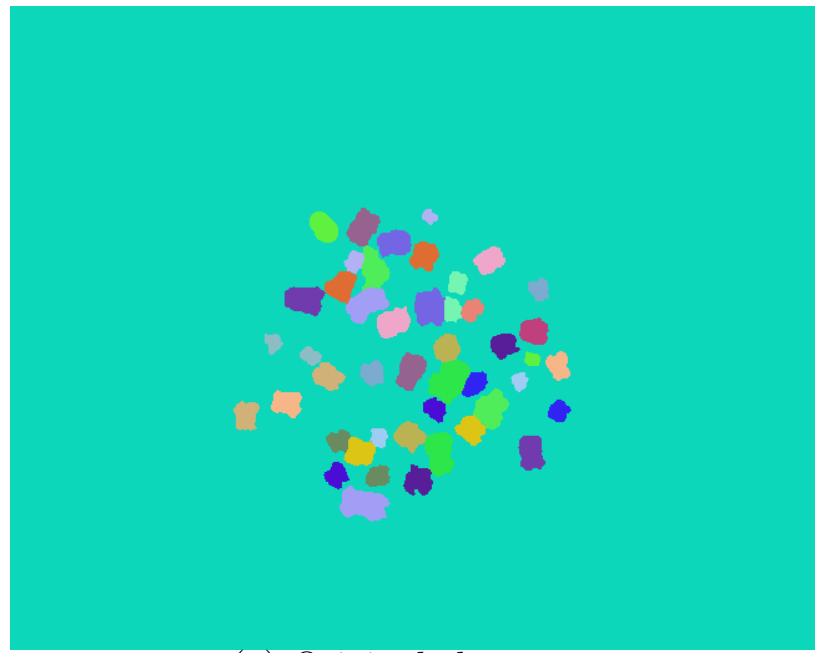

(a) Original class-map
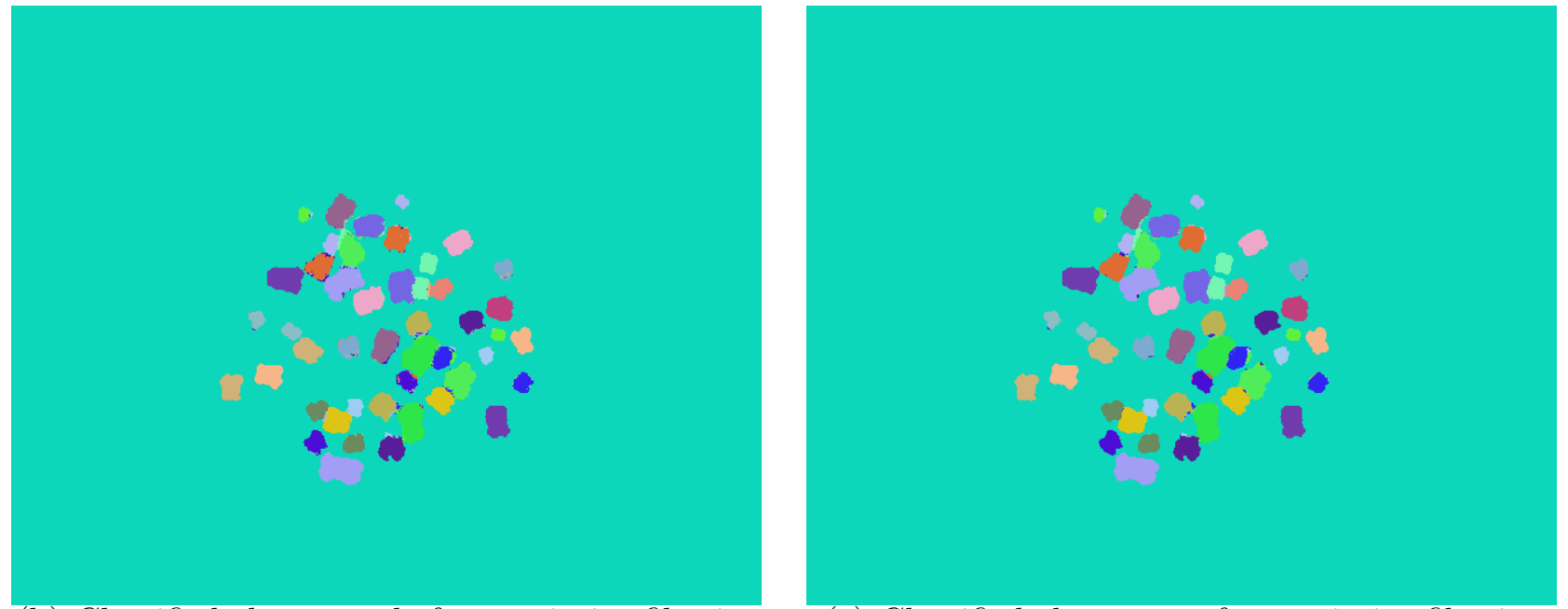

(b) Classified class-map before majority filtering

(c) Classified class-map after majority filtering

Fig. 4. Classification results for M-FISH Image Set B 


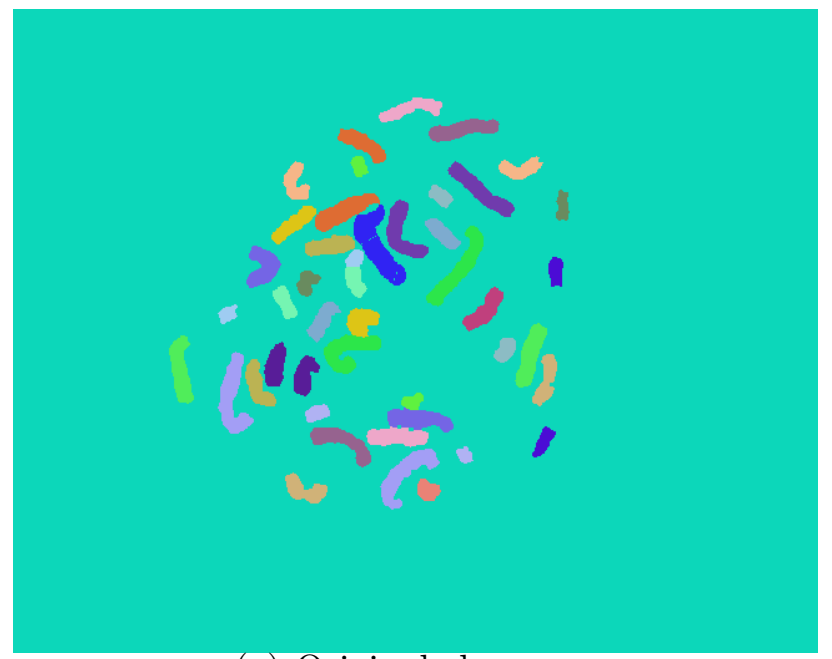

(a) Original class-map
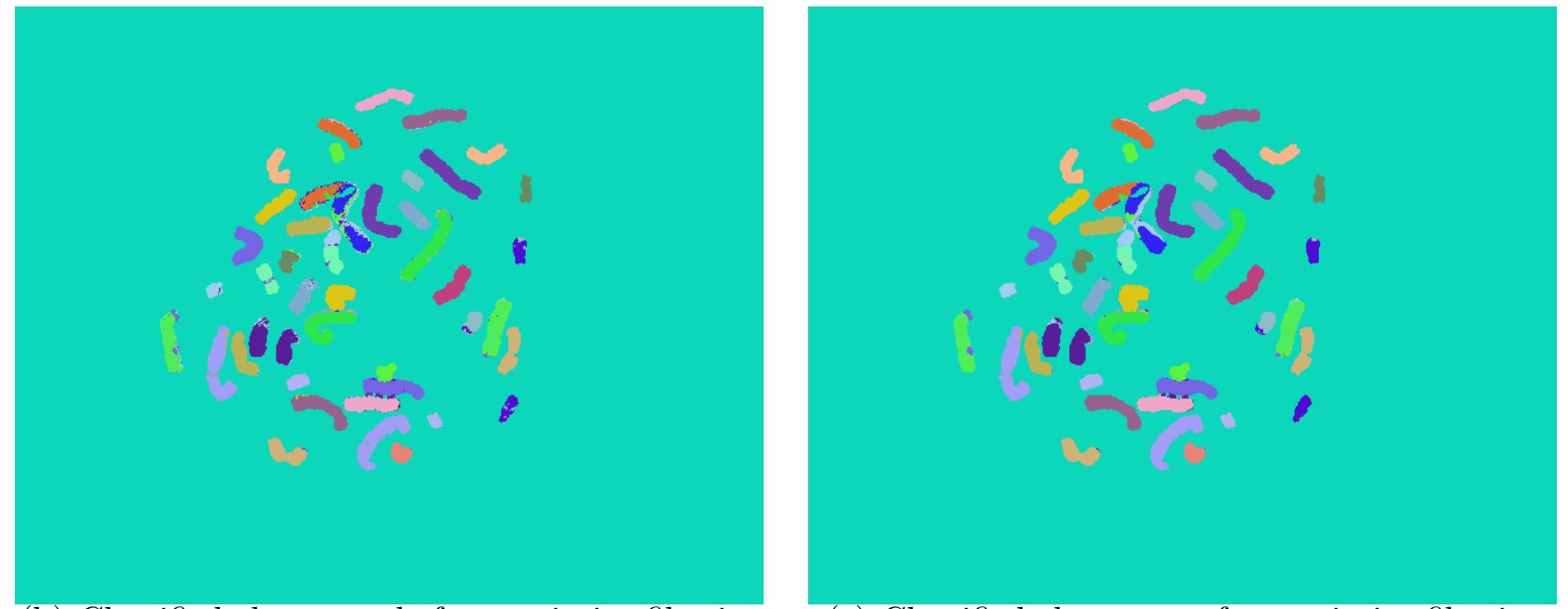

(b) Classified class-map before majority filtering

(c) Classified class-map after majority filtering

Fig. 5. Classification results for M-FISH Image Set C 


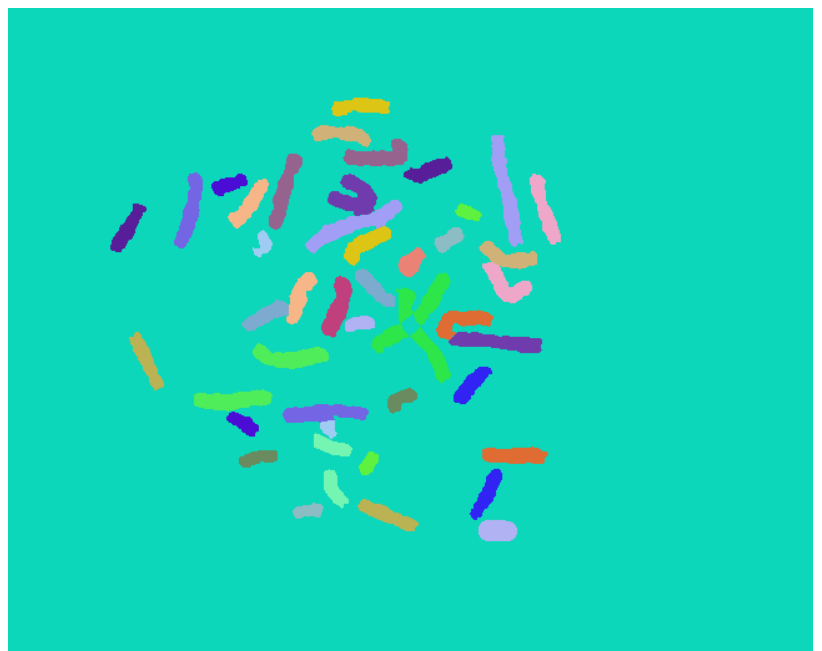

(a) Original class-map
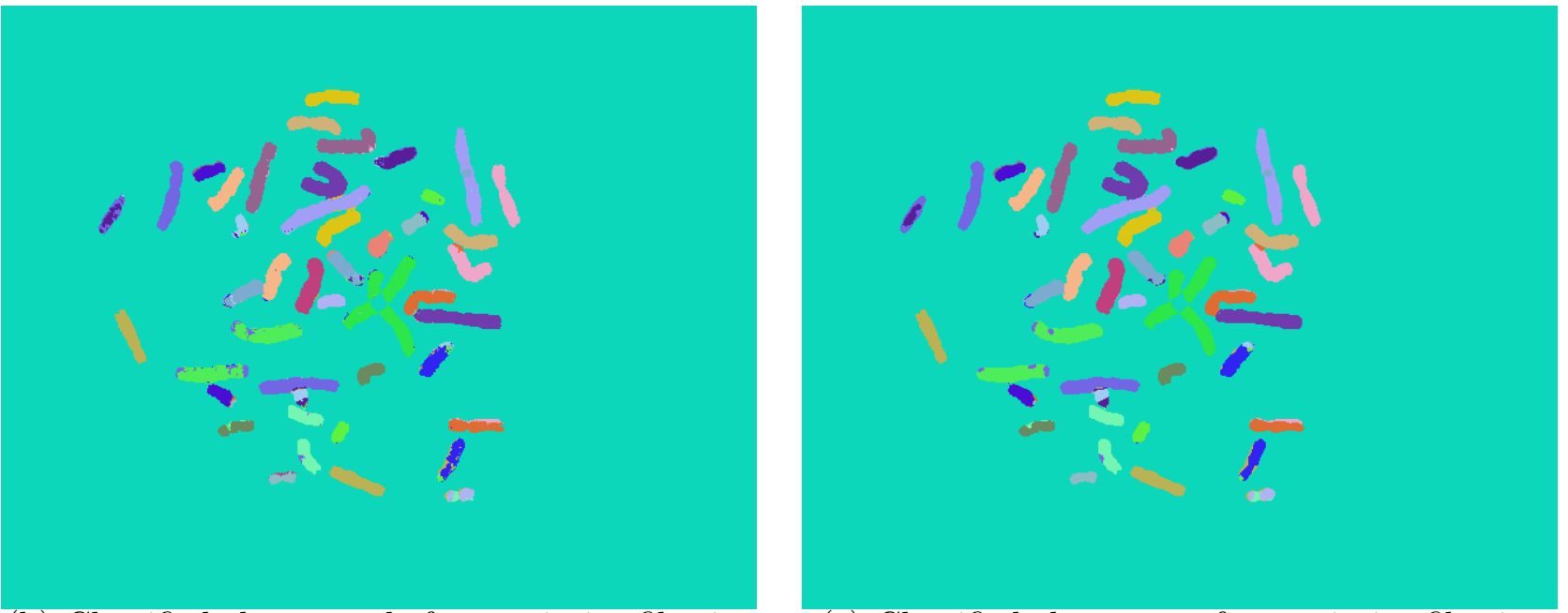

(b) Classified class-map before majority filtering

(c) Classified class-map after majority filtering

Fig. 6. Classification results for M-FISH Image Set D 


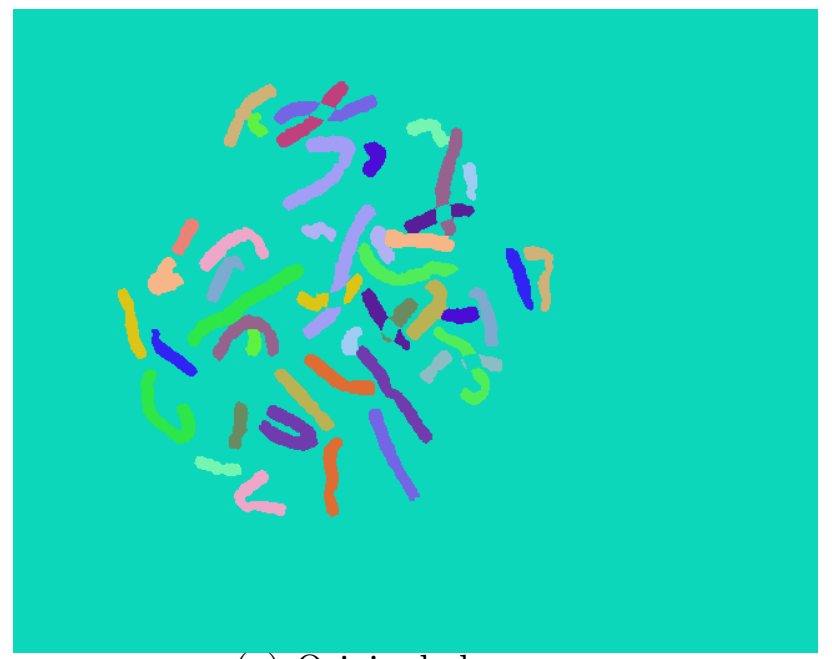

(a) Original class-map
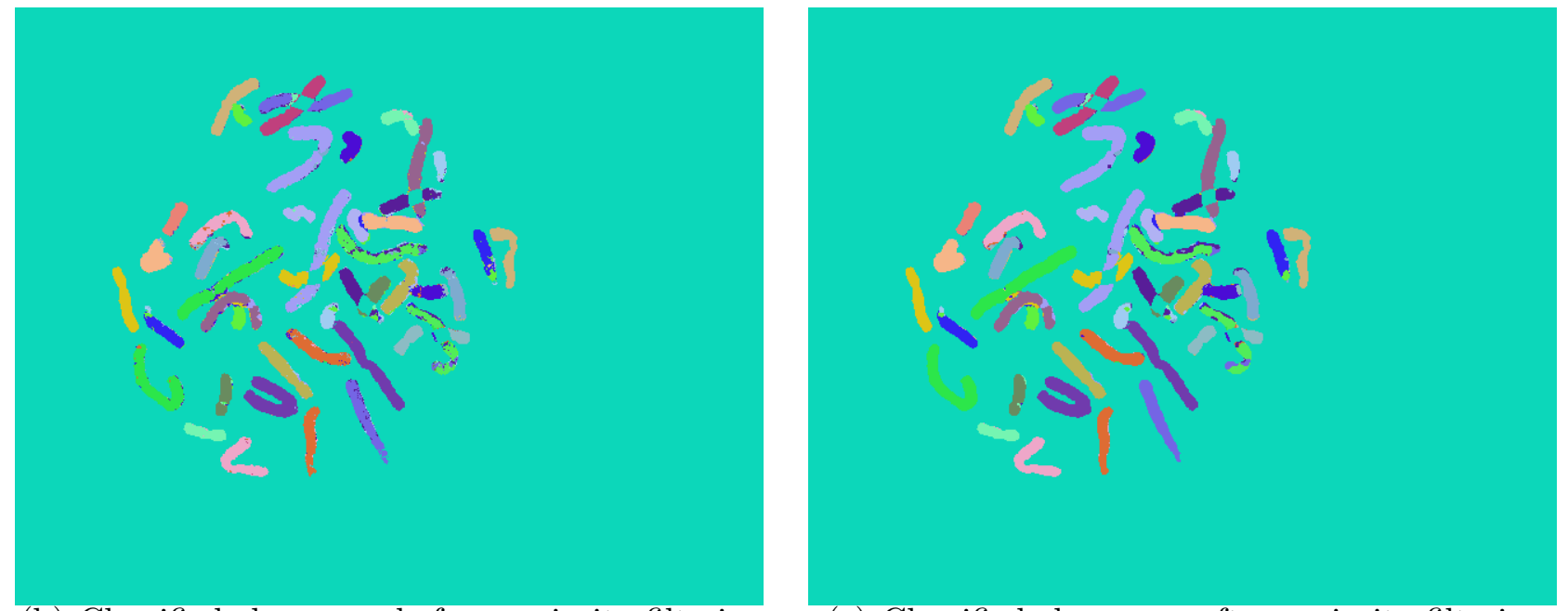

(b) Classified class-map before majority filtering

(c) Classified class-map after majority filtering

Fig. 7. Classification results for M-FISH Image Set E 


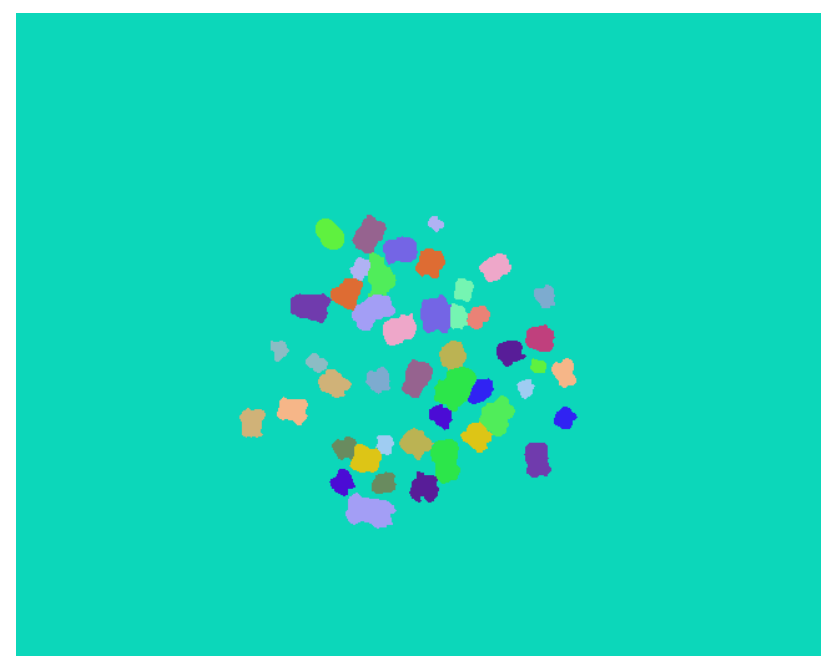

(a) Original class-map

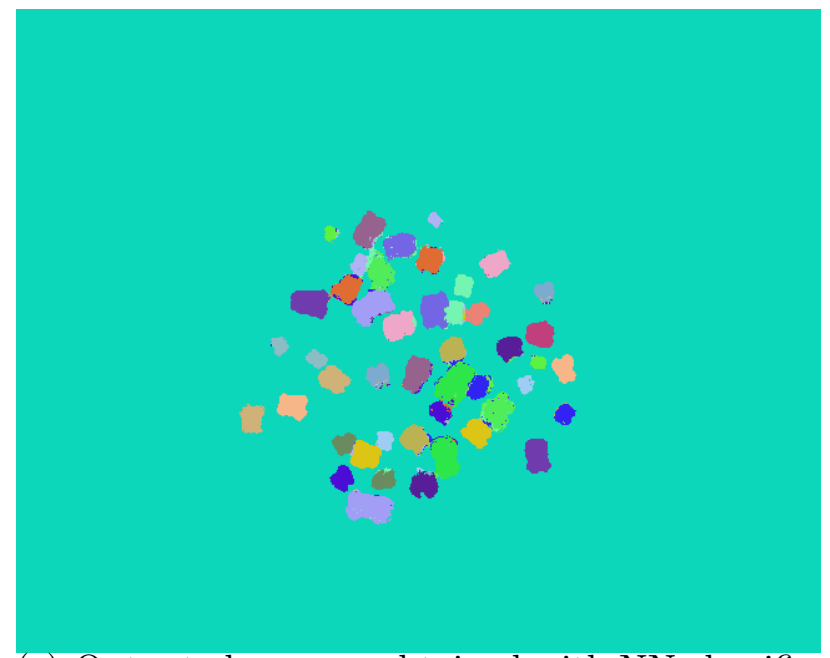

(c) Output class-map obtained with NN classifier

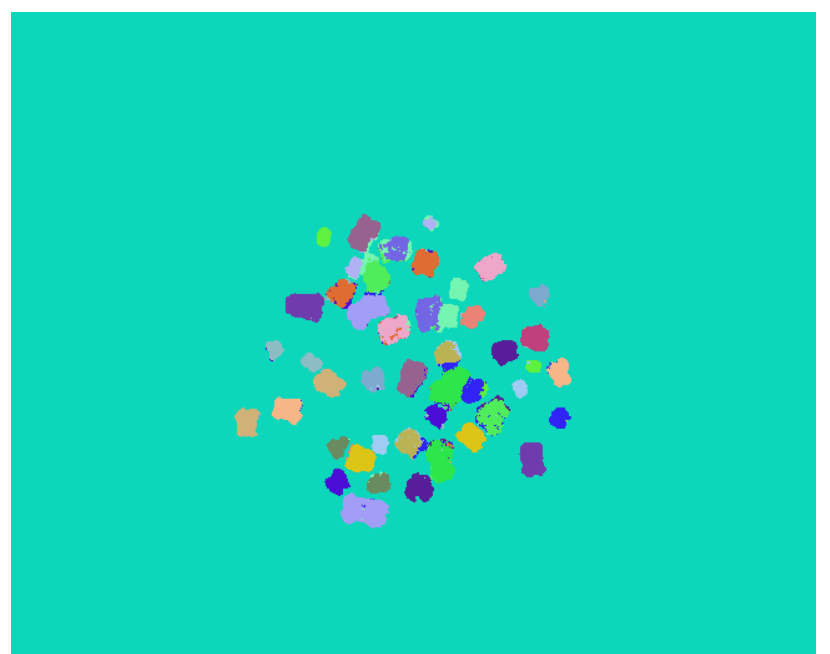

(b) Output class-map obtained with MLE classifier

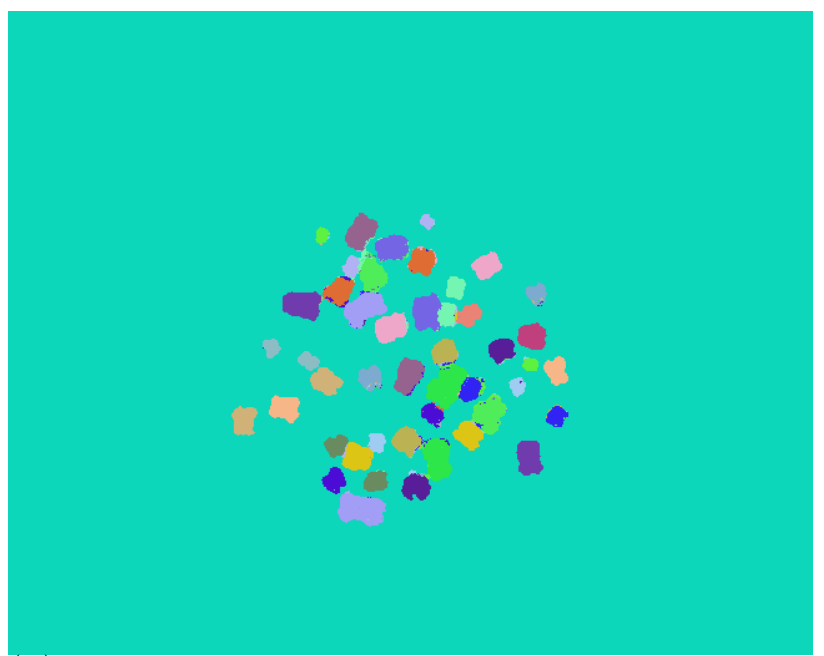

(d) Output class-map obtained with k-NN classifier $(\mathrm{k}=7)$

Fig. 8. The different classification results obtained with the MLE, NN and k-NN (k-7) classifiers, for M-FISH Image Set B 